\title{
Upcoming direct acting antivirals for hepatitis $C$ patients with a prior treatment failure
}

\author{
Tommaso Lorenzo Parigi ${ }^{1,2}$, Maria Corina Plaz Torres', and Alessio Aghemo ${ }^{1,2}$ \\ 'Division of Internal Medicine and Hepatology, Humanitas Research Hospital IRCCS, Rozzano; ${ }^{2}$ Department of Biomedical Sciences, \\ Humanitas University, Pieve Emanuele, Italy
}

Despite the high efficacy of direct acting antivirals (DAAs) not all patients successfully clear hepatitis $C$ virus infection, in fact, approximately $1-3 \%$ fail to reach a sustained virological response 12 weeks after end of treatment. DAA failures are characterized by advanced liver disease, specific genotypes/subtypes and resistance associated substitutions to the DAA class they have been treated with. Current European Association for the Study of the Liver guidelines recommend three therapeutic options for such patients. The first is a 12 week course of sofosbuvir (SOF), velpatasvir (VEL) and voxilaprevir (VOX), which has shown to be effective in $90-99 \%$ of patients and was granted A1 level recommendation. The second option, reserved for patients who have predictors of failure consists in 12 weeks regimen with glecaprevir (GLE) and pibrentasvir (PIB), effective in 90-97\%. Finally, although not supported by published data, for especially difficult to treat patients there should theoretically be a benefit in prolonged combinations of SOF+GLE/PIB or SOF/VEL/VOX tribavirin. This review presents the latest evidence from both clinical trials and real-life on such therapeutic strategies. (Clin Mol Hepatol 2019;25:360-365)

Keywords: Hepatitis C virus (HCV); Antivirals; Re-treatment; Sofosbuvir

\section{INTRODUCTION}

The characterization of the hepatitis C virus (HCV) life cycle coupled with the development of in vitro replication models of HCV has allowed the discovery of compounds that inhibit HCV replication, the direct acting antivirals (DAAs).' DAAs can be divided into 3 drug classes based on the HCV protein they target and block. Protease inhibitors (PI) target the NS3 protease which is essential for polyprotein processing and cleavage to release the structural and non structural (NS) HCV proteins, these drugs are characterized by the -previr suffix (glecaprevir [GLE], grazoprevir
[GRZ], voxilaprevir [VOX]). Polymerase inhibitors can be divided into nucleoside and non-nucleoside polymerase inhibitors based on the mechanisms which lead to inhibition of the NS5B RNA dependent polymerase. Nucleoside inhibitors include sofosbuvir (SOF), a potent uridine analogue, which acts as a RNA chain terminator during viral RNA synthesis, Non-nucleoside inhibitors include dasabuvir which blocks the NS5B polymerase through allosteric inhibition. This class of compounds is characterized by the -buvir suffix. The last class of DAAs are the NS5A inhibitors which target the NS5A replication complex, a protein without antiviral activity that is key to replication and viral release through stabili-

\footnotetext{
Abbreviations:

DAA, direct acting antiviral; DCV, daclatasvir; EBR, elbasvir; GLE, glecaprevir; GRZ, grazoprevir; HCV, hepatitis C virus; LDV, ledipasvir; NUCs, nucleotide inhibitor; PI, protease inhibitor; PIB, pibrentasvir; RASs, resistant associated substitutions; RBV, ribavirin; SOF, sofosbuvir; SVR, sustained virological response rates; VEL, velpatasvir; VOX, voxilaprevir
}

\section{Corresponding author : Alessio Aghemo}

Division of Internal Medicine and Hepatology, Humanitas Research Hospital IRCCS, Via Alessandro Manzoni, 56, Rozzano 20089, Italy

Tel: +39-282247006, Fax: +39-282242298

E-mail: Alessio.aghemo@hunimed.eu

https://orcid.org/0000-0003-0941-3226 
Tommaso Lorenzo Parigi, et al.

zation and protection from degradation of the HCV replication process. These NS5A inhibitors are characterized by the suffix -asvir (ledipasvir [LDV], velpatasvir [VEL], pibrentasvir [PIB], elbasvir [EBR]). The DAAs vary in terms of potency (velocity of HCV RNA decrease), barrier to resistance (ability to avoid selection of resistant associated viral substitutions) and genotypic activity. ${ }^{2}$ While the first 2 point can be improved by the combination of multiple DAAs, genotypic activity varies among the DAAs, with VEL, SOF, VOX, daclatasvir (DCV), GLE and PIB having pangenotypic activity while GRZ, EBR, LDV, paritaprevir, ombitasvir and dasabuvir having HCV genotype specific activity. To achieve optimal sustained virological response rates (SVR), the marker of HCV cure, DAAs need to be combined. Two possible strategies exist: first using SOF as the back bone and combining it with an NS5A inhibitor (SOF/VEL, SOF/DCV, SOF/LDV) or it with an NS5A inhibitor and a $\mathrm{PI}$ (SOF/VEL/VOX). The second strategy combines a PI plus an NS5A inhibitor and does not include SOF (GLE/PIB or GRZ/EBR). All these combinations when used properly are able to achieve SVR rates which top the $95 \%$ mark. ${ }^{3}$ Re-treatment of patients who fail first-line DAA treatment is more challenging and will be the focus of this review article.

\section{CHARACTERISTICS OF PATIENTS WHO FAILED A DAA COURSE}

Failure to DAA treatment is rare, this has been recently confirmed by several real-life cohorts which reported SVR rates in the $97-99 \%$ range. ${ }^{4}$ From a public health point of view this small percentage translates to a relatively large number of people, with estimates in the European region reporting that in 2020 nearly $47,000 \mathrm{HCV}$ patients will have failed first-line DAA therapy. As highlighted by a recent analysis of the observational TARGET cohort, DAA treatment failures are most likely to be males with advanced fibrosis/cirrhosis and with signs of impaired liver function. A group of people at high risk of development of liver disease, hence in need of effective re-treatment strategies. In areas where genotype dependent DAA regimens have been widely used, treatment failures will be characterized by subtypes who show reduced susceptibility to DAA combinations such as HCV-1a in the USA, HCV-6c-i in Eastern Asia and genotype 4r in Europe and Sub Saharian Africa. ${ }^{5,6}$ These HCV strains are characterized by high rate of naturally occurring resistant associated substitutions (RASs) in the NS5A, NS3 and NS5B region which reduce the activity of genotype dependent SOF based regimens. On the other hand, these subtypes do not seem to play a major role in limiting the activity of pangenotypic DAA combinations.

DAA treatment failures are also characterized by prevalence of RAS in the region targeted by the received DAAs. Due to the high replication rate of HCV and the lack of proof reading activity of the NS5B polymerase, HCV genomes which include RASs are continuously generated. ${ }^{2}$ While the naturally occurring RASs, i.e., not selected by pharmacological pressure, are usually found in a minority of circulating $\mathrm{HCV}$ virions and thus do not affect treatment efficacy, the RAS that have been selected by treatment failure can become present in the majority of the circulating HCV quasispecies hence reducing the efficacy of re-treatment with the same DAA class. When patients who failed a DAA treatment undergo viral sequencing at the time of failure, around $84 \%$ of them will show class and drug specific RASs. This prevalence is higher in those who have received an NS5A regimen where nearly $95 \%$ of them will have RASs, and lower in those who have received a PI (64\%). RASs in the NS5B region that limit the efficacy of nucleotide inhibitors (NUCS) such as SOF are extremely difficult to detect, as these variants usually have low viral fitness and replicate very poorly. If patients who failed DAA treatment undergo regular follow-up with viral sequencing, NS5A RASs will persist for up to 2 years in nearly $90 \%$ of the cases. NS3 RASs, on the other hand, will revert to wild type in more than $80 \%$ of the cases after 1 year of follow-up.

\section{PRINCIPLES GUIDING RE-TREATMENT IN DAA FAILURES}

As previously discussed DAA failures are characterized by advanced liver disease, specific genotypes/subtypes and RASs to the DAA class they have failed. For this reason, the principles guiding the re-treatment of these patients differ from those used in firstline treatment. Although precise pre-treatment assessment to exclude significant drug-drug interactions and identify conditions which make DAAs unsafe (decompensated diseases) are still mandatory, most treatment recommendations suggest RAS testing to guide re-treatment. ${ }^{8-10}$ While this has yet to be proven cost effective or just effective compared to re-treatment without RAS testing, it is currently used to select "reinforced" re-treatment options. Re-treatment should ideally be based on SOF, as this drug has the highest genetic barrier to resistance of all available DAAs, and should avoid re-treating patients with the same DAA classes that have led to treatment failure. Ribavirin (RBV), which has been 
abandoned as a first-line treatment option with currently available and approved DAA combinations, can still be considered in particularly difficult patients. However, RBV use has to be weighed against the increase in side effects and the impairment in quality of life associated with its use and the lack of strong data supporting its use. ${ }^{11,12}$ Indeed, most recommendations on re-treatment strategies do not stem from clinical trials but should rather be seen as expert opinions.

\section{TREATMENT OPTIONS FOR DAA FAILURES}

According to European Association for the Study of the Liver (EASL) 2018 Guidelines, ${ }^{9}$ there are three options for HCV infected patients who previously failed after a course of DAA.

\section{First-line treatment}

The first-line for re-treatment is a 12 weeks SOF/VEL/VOX regimen, and the evidence for such indication was initially based on the POLARIS 1 and 4 trials. ${ }^{13}$

POLARIS 1, a phase III multicenter randomized clinical trial, proved both safety and efficacy of SOF/VEL/VOX 12 weeks regimen for patients previously treated with NS5A inhibitors. Among the 263 patients included in the active arm, all genotypes 1 to 6 were represented and 46\% (121) had compensated Child-Pugh A cirrhosis. The overall SVR rate was 96\%, 99\% in the non-cirrhotic subgroup and 93\% among those with cirrhosis. Of the 263 ac- tively treated patients 10 did not reach a sustained virological response, of whom 7 had a virological failure, 2 withdrew consent and 1 was lost to follow-up.

The POLARIS 4 trial confirmed the superiority of the triple therapy with SOF/VEL/VOX versus a double drug regimen with SOF/ VEL. In particular, 333 patients who had been previously treated with any DAA regimen except NS5A inhibitors were enrolled and treated; 163 were randomly assigned to receive SOF/VEL/VOX 12 weeks and 151 were assigned to the SOF/VEL same duration arm. The SVR12 rate among the SOF/VEL/VOX arm was 98\%, significantly superior to the $90 \%$ of the SOF/VEL group (Fig. 1). Table 1 shows a recap of the phase III randomized clinical trials on SOF/ VEL/VOX.

Additional real-life data, mainly from the US, confirmed high rates of SVR12 in experienced populations treated with 12 weeks SOF/VEL/VOX \pm RBV. Saxena et al. ${ }^{14}$ reported 97\% SVR (208/214) in an experienced population (89\% and 94\% NS5A and NS5B inhibitors respectively), the TRIO Network ${ }^{15}$ showed a 99\% (163/165) in a similarly experienced group, finally Belperio and colleagues reported SVR rates greater than $90 \%$ ( $\geq 95 \%$ for genotype 1 [GT1], and $\geq 93 \%$ among GT3) in a real-world cohort of 573 non-naive patients. ${ }^{16}$ Interesting in the study by Belperio et al. ${ }^{16}$, when analyzing patients who previously failed SOF/VEL, SVR rates dropped to $82 \%, 86 \%$ and $85 \%$ for genotype 1,2 and 3 (Table 2).

\section{Second-line treatment}

For patients infected who previously failed with at least one

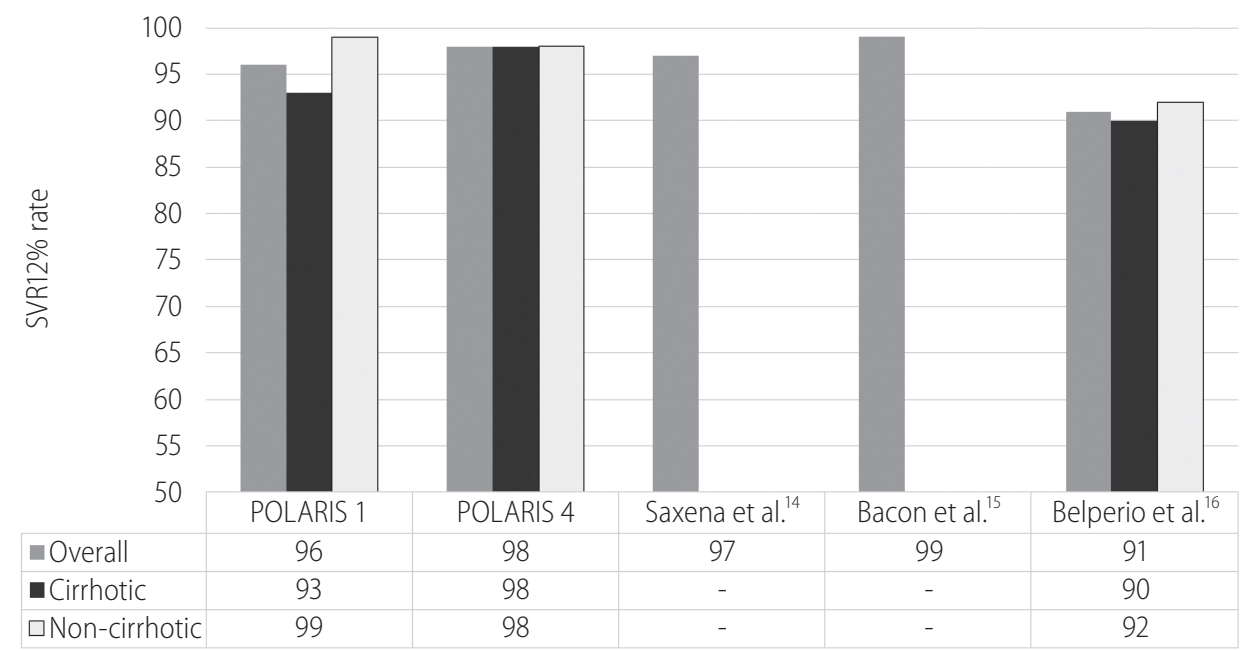

Figure 1. Sustained virological response (SVR) rates to sofosbuvir/velapatasvir/voxilaprevir in previous direct acting antiviral fauilures. Sofosbuvir/velpatasvir/voxilaprevir for the re-treatment of direct acting antiviral failures. SVR12, sustained virological response at week 12. 
Tommaso Lorenzo Parigi, et al.

Table 1. Registration Trials of sofosbuvir (SOF)/velpatasvir (VEL)/voxilaprevir (VOX)

\begin{tabular}{|c|c|c|}
\hline Trial & POLARIS 1 & POLARIS 4 \\
\hline Participants & 263 vs. 152 (placebo arm) & 333 (182 vs. 151) \\
\hline Design & SOF/VEL/VOX vs. placebo & SOF/VEL/VOX vs. SOF/VEL \\
\hline Duration & 12 weeks & 12 weeks \\
\hline Genotypes & $1-6$ & $1-4$ \\
\hline Compensated cirrhosis & $121(46 \%)^{*}$ in the active arm & $153(46 \%)$ \\
\hline Previous DAA experience & $\begin{array}{l}\text {-NS5A inhibitor+NS3 inhibitor+NS5B inhibitor: } 83 \text { (32\%) } \\
\text {-NS5A inhibitor+NS5B inhibitor } 161 \text { (61\%) } \\
\text {-NS5A inhibitor } 18 \text { (7\%) }\end{array}$ & $\begin{array}{l}\text {-NS5B inhibitor+NS3 inhibitor } 46 \text { (25\%) } \\
\text {-NS5B inhibitor } 134 \text { (74\%) } \\
\text {-SOF/VEL/VOX arm* }\end{array}$ \\
\hline Overall SVR12 rate & $96 \%(253 / 263)$ & $98 \%(178 / 182)$ vs. $90 \%(136 / 151)$ \\
\hline SVR per single GT & $\begin{array}{c}\text { GT1 97\% (146/150) } \\
\text { GT1a 96\% (97/101) } \\
\text { GT1b 100\% (45/45) } \\
\text { GT2 5/5 } \\
\text { GT3 95\% (74/78) } \\
\text { GT4 } 91 \%(20 / 22) \\
\text { GT5 1/1 } \\
\text { GT6 6/6 }\end{array}$ & $\begin{array}{l}\text { GT1a 98\% (53/54) } \\
\text { GT1b 96\% (23/24) } \\
\text { GT2 100\% (31/31) } \\
\text { GT3 96\% (52/54) } \\
\text { GT4 100\% (19/19) } \\
\text { SOF/VEL/VOX arm* }\end{array}$ \\
\hline
\end{tabular}

DAA, direct acting antiviral; SVR12, sustained virological response (SVR) at week 12; GT, genotype.

*SOF/VEL/VOX Arm as written on the right.

Table 2. Sofosbuvir (SOF)/velpatasvir (VEL)/voxilaprevir (VOX) efficacy real-life data

\begin{tabular}{|c|c|c|c|}
\hline & \multicolumn{3}{|c|}{ Study-first author } \\
\hline & $\begin{array}{l}\text { Kaiser Permanente- } \\
\text { Saxena et al. }{ }^{14}\end{array}$ & $\begin{array}{l}\text { TRIO Network- } \\
\text { Bacon et al. }{ }^{15}\end{array}$ & $\begin{array}{l}\text { Veteran Administration- } \\
\text { Belperio et al. }{ }^{16}\end{array}$ \\
\hline Treatment regimen & 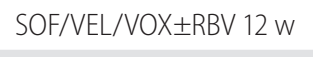 & SOF/VEL/VOX \pm RBV $12 \mathrm{w}$ & SOF/VEL/VOX $12 \mathrm{w}$ \\
\hline DAA experience in the overall cohort & $85 \%$ & $88 \%$ & $100 \%$ \\
\hline SVR 12 among experienced only & $97 \%(208 / 214)$ & $99 \%(163 / 165)$ & $91 \%(501 / 551)$ \\
\hline
\end{tabular}

RBV, ribavirin; w, weeks; DAA, direct acting antiviral; SVR12, sustained virological response at week 12.

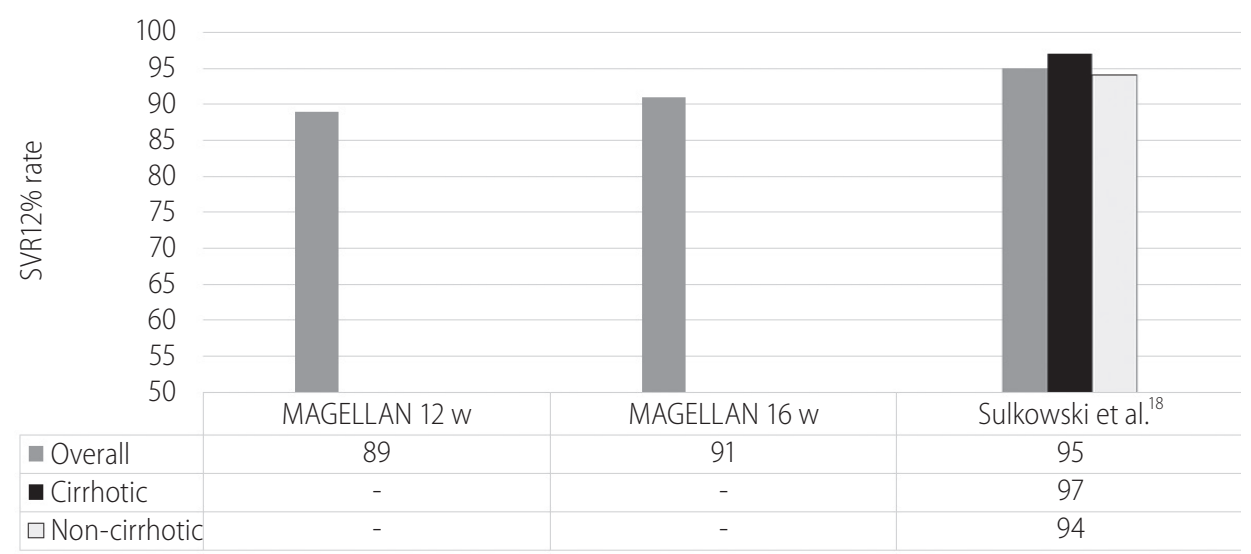

Figure 2. SVR rates following re-treatment of direct acting antiviral (DAA) failures with glecaprevir/pibrentasvir. Glecaprevir/pibrentasvir for the retreatment of DAA failures. SVR12, sustained virological response at week 12; w, weeks. 
NS3/4A protease and/or NS5A inhibitor+SOF, and have predictors of poor response such as: advanced liver disease, received multiple courses of DAA and or have complex RAS profile, EASL 2018 guidelines recommend a 12 weeks $\mathrm{SOF}+\mathrm{GLE} / \mathrm{PIB}$ regimen (B2). ${ }^{9}$

Data supporting the use of GLE/PIB came from the MAGELLAN-1 (part 2) study, ${ }^{17}$ where a cohort of 91 patients (GT1 and GT4, 30\% cirrhotic) were randomized 1:1 to receive either 12 or 16 weeks of GLE/PIB. Results showed a SVR12 rate of $89 \%$ for the group treated with the short regimen, and $91 \%$ for those who received 16 weeks. GLE/PIB was particularly effective among patients naïve or with single DAA experience while SVR rates among those previously treated with both NS5A and NS3/4A inhibitors was suboptimal, possibly due to a low barrier to resistance of GLE/PIB. Thus, the indication for the SOF add-on.

Real-life data by Sulkowski and colleagues ${ }^{18}$ reported a $95 \%$ SVR12 rate in participants with GT1 infection (94\% for non-cirrhotic and $97 \%$ for cirrhotic, respectively), hence supporting the use of 16 weeks GLE/PIB regimen for re-treatment of prior NS5Ai/ SOF \pm RBV GT 1 regardless of the presence of cirrhosis (Fig. 2).

\section{Third-line treatment}

Finally, the third EASL 2018 treatment recommendation suggests the possibility of extending treatment duration for either SOF/VEL/VOX or SOF/GLE/PIB, both with or without RBV, in particularly difficult cases, namely patients with NS5A RASs who failed twice to achieve SVR after a combination regimen including a protease and/or an NS5A inhibitor. However, this recommendation is not supported by published data and such decision should be taken by expert multidisciplinary teams for each patient in consideration of his clinical condition and virological profile.

\section{Re-treatment of patients with decompensated cirrhosis}

All the EASL endorsed regimens for the re-treatment of DAA failures are based on a PI. PIs are metabolized in the liver and excreted in bile. For this reason pharmacokinetic properties of PIs are influenced by the degree of liver function impairment. Patients with decompensated cirrhosis (Child Pugh B and C) have reduced activity of the CYP3a4 enzyme family, have spontaneous intra hepatic shunts which decrease the quantity of drug which is metabolized or excreted by the liver and also have reduced drug protein binding due to reduced albumin synthesis. Thus when given in volunteers with Child Pugh (CPT) B and C cirrhosis, Pls show an increase in plasma concentration of 5 and 12 times respectively. ${ }^{19}$ This increase in Pls concentration is associated with significant side effects as shown by several studies reporting cases of death, sepsis and liver transplantation. ${ }^{17,20}$ For this reason Pls are contraindicated in CPT B and $C$ and should be managed with caution in CPT A6 ${ }^{9}$ (EASL 2018). The efficacy of SOF/VEL given for 24 weeks plus RBV was studied in 69 patients who had previously failed a DAA (59\% failed SOF/VEL and 39\% failed SOF/VEL/VOX). ${ }^{21}$ Overall the SVR rate was $91 \%$, with similarly high SVR rates in patients with HCV-1 (97\%) and HCV-2 (93\%). On the other hand, SVR rates were suboptimal in HCV-3 patients (78\%). This was due to the presence of RASs in the NS5A region, in the $76 \%$ of enrolled HCV-3 patients who showed NS5A RASs the overall SVR dropped to $77 \%$. In HCV-1 and 2 the presence of baseline RASs did not have such a dramatic effect, as the SVR was 100\% and $89 \%$ respectively. The study findings highlight that previous DAA failures with decompensated disease remain a group of patients where treatment efficacy is sub-optimal.

\section{CONCLUSIONS}

Evidence coming from clinical trials as well as real-life studies is gathering on the efficacy of DAAs in the re-treatment of previous failures. Efficacy of SOF/VEL/VOX is elevated across genotypes and stages of disease severity, although some real-life cohorts reported suboptimal results in those who had previously failed SOF/ VEL. GLE/PIB given for 16 weeks has demonstrated high SVR rates although in a relatively small study. The combination of SOF+GLE/PIB or SOF/VEL/VOX+RBV have been understudied, but in theory should provide patients with the best SVR rate even in the presence of multiple predictive factors of failure. All these regimens include a PI, a drug class which is still contraindicated in patients with decompensated cirrhosis due to increased risk of side effects, liver function deterioration and death. In patients with decompensated cirrhosis the current recommended regimen is the combination of SOF/VEL+RBV for 24 weeks, a regimen which might achieve suboptimal SVR rates in those with baseline NS5A RASs. This highlights the need for quick access to first-line DAA treatment and to re-treatment options to avoid progression of liver disease to decompensated cirrhosis and lack of effective DAA combinations.

\section{Author's contributions}

All authors have contributed to data retrieval, analysis and draft 
Tommaso Lorenzo Parigi, et al.

of the manuscript.

\section{Conflicts of Interest}

TLP, MCPT have nothing to discolose.

$A A$ has received research grants from Gilead and Abbvie, and is an advisor for MSD, Gilead, Abbvie and Intercept.

\section{REFERENCES}

1. Aghemo A, De Francesco R. New horizons in hepatitis $C$ antiviral therapy with direct-acting antivirals. Hepatology 2013;58:428-438.

2. Pawlotsky JM. Hepatitis C virus resistance to direct-acting antiviral drugs in interferon-free regimens. Gastroenterology 2016;151:70-86.

3. D'Ambrosio R, Degasperi E, Colombo M, Aghemo A. Direct-acting antivirals: the endgame for hepatitis C? Curr Opin Virol 2017;24:31-37.

4. D'Ambrosio R, Pasulo L, Puoti M, Vinci M, Schiavini M, Lazzaroni S, et al. Real-world effectiveness and safety of glecaprevir/pibrentasvir in 723 patients with chronic hepatitis C. J Hepatol 2019;70:379-387.

5. Mettikanont P, Bunchorntavakul C, Reddy KR. Systematic review: epidemiology and response to direct-acting antiviral therapy in genotype 6 chronic hepatitis C virus infection. Aliment Pharmacol Ther 2019:49:492-505.

6. Fourati S, Rodriguez C, Hézode C, Soulier A, Ruiz I, Poiteau L, et al. Frequent antiviral treatment failures in patients infected with hepatitis C virus genotype 4, subtype 4r. Hepatology 2019;69:513-523.

7. Jeong $Y$, Jin B, Lee HW, Park HJ, Park JY, Kim DY, et al. Evolution and persistence of resistance-associated substitutions of hepatitis C virus after direct-acting antiviral treatment failures. J Viral Hepat 2018;25:1251-1259.

8. Aghemo A, Piroth L, Bhagani S. What do clinicians need to watch for with direct-acting antiviral therapy? J Int AIDS Soc 2018;21 Suppl 2:e25076

9. European Association for the Study of the Liver. EASL recommendations on treatment of hepatitis C 2018. J Hepatol 2018;69:461-511.

10. AASLD-IDSA HCV Guidance Panel. Hepatitis C guidance 2018 update: AASLD-IDSA recommendations for testing, managing, and treating hepatitis C virus infection. Clin Infect Dis 2018;67:14771492.

11. Younossi ZM, Stepanova M, Charlton M, Curry MP, O'Leary JG, Brown RS, et al. Patient-reported outcomes with sofosbuvir and velpatasvir with or without ribavirin for hepatitis C virus-related decompensated cirrhosis: an exploratory analysis from the ran- domised, open-label ASTRAL-4 phase 3 trial. Lancet Gastroenterol Hepatol 2016;1:122-132.

12. Takehara T, Sakamoto N, Nishiguchi S, Ikeda F, Tatsumi T, Ueno Y, et al. Efficacy and safety of sofosbuvir-velpatasvir with or without ribavirin in HCV-infected Japanese patients with decompensated cirrhosis: an open-label phase 3 trial. J Gastroenterol 2019;54:87-95.

13. Bourlière M, Gordon SC, Flamm SL, Cooper CL, Ramji A, Tong M, et al. Sofosbuvir, velpatasvir, and voxilaprevir for previously treated HCV infection. N Engl J Med 2017;376:2134-2146.

14. Saxena V, Chamberland S, Hurley L, Lai JB, Truong E, Marcus J, et al. Real-world safety and effectiveness of sofosbuvir/velpatasvir/voxilaprevir and glecaprevir/pibrentasvir in hepatitis $C$ infected patients [Abstract]. Hepatology 2018;68:418A-419A.

15. Bacon BR, Curry MP, Flamm SL, Milligan S, Tsai N, Wick N, et al. Sofosbuvir/velpatasvir/voxilaprevir (SOF/VEL/VOX) in care of chronic hepatitis C patients; clinical practice experience from the TRIO network [Abstract]. Hepatology 2018;68:419A.

16. Belperio P, Bakckus L, Shahoumian T, Loomis T, Mole L. Real-world effectiveness of sofosbuvir/velpatasvir/voxilaprevir in 573 treatmentexperienced patients with hepatitis C [Abstract]. Hepatology 2018;68(Suppl 1):S227.

17. Poordad F, Felizarta F, Asatryan A, Sulkowski MS, Reindollar RW, Landis CS, et al. Glecaprevir and pibrentasvir for 12 weeks for hepatitis $C$ virus genotype 1 infection and prior direct-acting antiviral treatment. Hepatology 2017;66:389-397.

18. Sulkowski MS, Lok AS, Willner IK, Reddy KR, Shiffman ML, Hassan MA, et al. High efficacy of glecaprevir/pibrentasvir in patients with chronic HCV GT1 infection who failed prior treatment with NS5A-inhibitor plus sofosbuvir regimens [Abstract]. Hepatology 2018:68:141A-142A.

19. Smolders EJ, de Kanter CT, van Hoek B, Arends JE, Drenth JP, Burger DM. Pharmacokinetics, efficacy, and safety of hepatitis $C$ virus drugs in patients with liver and/or renal impairment. Drug Saf 2016;39:589-611.

20. Petta S, Marzioni M, Russo P, Aghemo A, Alberti A, Ascione A, et al. Ombitasvir, paritaprevir, and ritonavir, with or without dasabuvir, plus ribavirin for patients with hepatitis C virus genotype 1 or 4 infection with cirrhosis (ABACUS): a prospective observational study. Lancet Gastroenterol Hepatol 2017;2:427-434.

21. Gane EJ, Shiffman ML, Etzkorn K, Morelli G, Stedman CAM, Davis $M N$, et al. Sofosbuvir-velpatasvir with ribavirin for 24 weeks in hepatitis $C$ virus patients previously treated with a direct-acting antiviral regimen. Hepatology 2017;66:1083-1089. 